\title{
Características produtivas de genótipos de cebola no Vale do São Francisco
}

\author{
Nivaldo D Costa ${ }^{1}$; Geraldo M de Resende ${ }^{1}$; Carlos AF Santos ${ }^{1}$; Wêydjane de Moura Leite ${ }^{2}$; José Maria Pinto ${ }^{1}$ \\ 'Embrapa Semi-Árido, C. Postal 23, 56302-970 Petrolina-PE; Bolsista DTI/CNPq; ndcosta@cpatsa.embrapa.br;
}

\section{RESUMO}

Com o objetivo de avaliar a produtividade de genótipos de cebola no Vale do São Francisco, foram conduzidos dois experimentos, de abril a agosto de 2003 e 2004, na Embrapa Semi-Árido, Petrolina-PE. Usou-se o delineamento experimental de blocos ao acaso, com quinze genótipos e quatro repetições e quatorze genótipos e três repetições, respectivamente em 2003 e 2004. Em 2003, a produtividade total de bulbos variou de 29,5 a $59,6 \mathrm{t} \mathrm{ha}^{-1}$ e a comercial oscilou de 18,6 a 51,7 $\mathrm{t} \mathrm{ha}^{-1}$. A massa fresca do bulbo variou de 104 a $186 \mathrm{~g}$ e os genótipos IPA-1 e Brisa apresentaram maior número de bulbos comerciais por parcela (113 bulbos). Em 2004, a produtividade total de bulbos variou de 30,4 a $58,4 \mathrm{t} \mathrm{ha}^{-1}$ e a comercial oscilou de 24,0 a 56,0 tha-1. A massa fresca do bulbo variou de 86 a 160 g e os genótipos EX-07593000, Granex 429 apresentaram maior número de bulbos comerciais por parcela (126 e 124 bulbos, respectivamente). Pelos resultados, em função das características avaliadas, os genótipos EX-19013, EX-07595002, Encino, IPA-11, Brisa, TPC 91923, TPR 91970 e Ex-075593000, em 2003, e os genótipos EX-0759300, Granex-429, IPA-11, IPA-04 e Texas Grano 502, em 2004, foram os mais adaptados e com maior potencial para cultivo no Vale do São Francisco.

Palavras-chave: Allium cepa, adaptação, competição, rendimento.

\begin{abstract}
Yield characteristics of onion genotypes in the São Francisco River Valley

Two field trials were carried out at Embrapa Semi-Árido, Petrolina, Brazil. The experiment was carried out from April to August of 2003 and 2004, in a randomized complete block design. In 2003, fifteen genotypes and four replications were used and in 2004, fourteen genotypes and three repetitions were used. In 2003, the total yield of bulbs varied from 29.5 to $59.6 \mathrm{t} \mathrm{ha}^{-1}$ and the commercial yield varied from 18.6 to $51.7 \mathrm{t} \mathrm{ha}^{-1}$. The fresh mass of bulbs varied from 104 to $186 \mathrm{~g}$ and the genotypes IPA-11and Brisa showed the highest number of commercial bulbs per plot (113 bulbs). In 2004, the total yield of bulbs varied from 30.4 to $58.4 \mathrm{t} \mathrm{ha}^{-1}$ and the commercial yield varied from 24.0 to $56.0 \mathrm{t} \mathrm{ha}^{-1}$. The fresh mass of bulbs varied from 86 to $160 \mathrm{~g}$ and the genotypes EX-07593000 and Granex 429 showed the highest number of commercial bulbs per plot (126 and 124 bulbs, respectively). Based on the evaluated characteristics, the genotypes EX-19013, EX-07595002, Encino, IPA-11, Brisa, TPC 91923, TPR 91970 and EX-075593000, in 2003, and the genotypes EX-0759300, Granex-429, IPA-11, IPA-04 and Texas Grano 502, in 2004, were the best adapted and with the highest potential for planting in the São Francisco River Valley.
\end{abstract}

Keywords: Allium cepa, adaptation, competition, yield.

(Recebido para publicação em 10 de julho de 2006; aceito em 9 de abril de 2007)

A cebola (Allium cepa L.), dentre as espécies oleracéas cultivadas pertencentes ao gênero Allium, é a mais importante sob o ponto de vista de volume de consumo e valor econômico (Souza $\&$ Resende, 2002). No Brasil, destacase ao lado da batata e do tomate como as mais importantes economicamente tanto pelo volume produzido, em torno de 1 milhão de $\mathrm{t}^{\mathrm{ano}} \mathrm{o}^{-1}$, como pela renda gerada. A cebolicultura nacional é uma atividade praticada principalmente por pequenos produtores e a sua importância sócio-econômica fundamenta-se não apenas em demandar grande quantidade de mão-de-obra, contribuindo na viabilização de pequenas propriedades, como, também, em fixar os pequenos produtores na zona rural, reduzindo desse modo a migração para as grandes cidades (Costa et al,, 2002).

A produção mundial em 2005 foi de 57,4 milhões de toneladas, cultivadas em área de 3,2 milhões de hectares, o que proporcionou uma produtividade média de 17,9 t ha $^{-1}$ (FAO, 2006). A produtividade média nacional em 2004 situou-se em 17,9 t ha-1, e nos estados de Pernambuco e Bahia, maiores produtores do Nordeste, se alcançou produtividade média de 21,2 e 24,3 $\mathrm{tha}^{-1}$, respectivamente (IBGE, 2005).

Há relatos de diferentes produtividades da cebola, de acordo com o local de produção. No sul de Minas Gerais, a produtividade de bulbos comerciais oscilou entre 22,6 a 50,2 t ha ${ }^{-1}$, destacando-se as cultivares Granex $429(50,2 \mathrm{t}$ ha $\left.^{-1}\right)$ e Texas Grano 502 (44,4 tha $\left.{ }^{-1}\right)$, com menores rendimentos obtidos pela cultivar Chata Roxa com 22,6 t ha-1 e variações de 46,0 a 97,7 g para a massa fresca do bulbo (Resende et al., 2003). Vidigal et al. (2002) informam produtividades de 13,3 a 32,9 $\mathrm{t} \mathrm{ha}^{-1}$ para o Norte de Minas Gerais. No Semi-Árido piauiense, As cultivares foram divididas em três grupos, de acordo com sua pro- dutividade: As cultivares do primeiro grupo (Vale Ouro IPA-11, Franciscana IPA-10 e Mercedes) apresentaram produtividade de bulbos comerciais oscilando de 40,5 a 43,7 t ha-1; No segundo grupo, as cultivares Princesa, Granex 33, Duquesa, Régia, Granex 429, Serrana, Composto IPA-6, Texas Grano 502 e Baia Periforme Precoce apresentaram produtividade variando de 38,9 a $34,6 \mathrm{t}$ $\mathrm{ha}^{-1}$; e no terceiro grupo as cultivares Piraouro, Linda Vista, Granex Ouro, Conquista, Crioula e Belém IPA-9, produziram 32,4 t/ha a 26,9 t/ha (Duarte et al., 2003).

Produtividades de bulbos comerciais variando entre 21,1 a 61,8 $\mathrm{tha}^{-1}$ para as condições do Vale do São Francisco são relatadas por Costa et al. (2000), que destacaram as cultivares Texas Grano PRR (61,8 t ha-1), Granex 429 (58,3 t $\left.\mathrm{ha}^{-1}\right)$, Texas Grano 438 (57,0 $\left.\mathrm{t} \mathrm{ha}^{-1}\right)$, Brownsville (55,4 $\left.\mathrm{t} \mathrm{ha}^{-1}\right)$, Texas Grano $502\left(53,9 \mathrm{tha}^{-1}\right)$ e Houston $\left(53,4 \mathrm{t} \mathrm{ha}^{-1}\right)$ 
Tabela 1. Produtividade total e comercial, massa fresca do bulbo e número de bulbos comerciais por parcela de genótipos de cebola (Commercial and total yield, fresh mass of bulbs and number of commercial bulbs in each parcel of onion genotypes). Petrolina, Embrapa Semi-Árido, 2004.

\begin{tabular}{|c|c|c|c|c|}
\hline \multirow{2}{*}{ Genótipos } & \multicolumn{2}{|c|}{ Produtividade (t ha-1) } & \multirow{2}{*}{$\begin{array}{l}\text { Massa fresca } \\
\text { de bulbos (g) }\end{array}$} & \multirow{2}{*}{$\begin{array}{c}\mathbf{N}^{\circ} \\
\text { bulbos/parcela }\end{array}$} \\
\hline & Total & Comercial & & \\
\hline TPR 91970 & 59,6 a & $45,2 \mathrm{a}$ & $177 \mathrm{a}$ & $91 \mathrm{a}$ \\
\hline EX-19013 & 58,6 a & 51,7 a & $186 a$ & 95 a \\
\hline EX-07595002 & 52,3 a & 48,6 a & 178 a & 99 a \\
\hline TPC 91923 & $52,2 \mathrm{a}$ & 45,5 a & $162 \mathrm{a}$ & $101 \mathrm{a}$ \\
\hline Encino & 51,6 a & 48,3 a & 176 a & 96 a \\
\hline EX-07593000 & $50,1 \mathrm{a}$ & $42,2 \mathrm{a}$ & $137 a$ & 97 a \\
\hline EX-07592000 & 48,3 a & $32,9 \mathrm{~b}$ & $149 a$ & $79 \mathrm{~b}$ \\
\hline IPA-11 & 47,7 a & 47,6 a & $137 \mathrm{a}$ & $113 a$ \\
\hline Brisa & 47,6 a & 46,6 a & $153 a$ & $113 a$ \\
\hline Superex & 46,3 a & $32,9 \mathrm{~b}$ & $146 a$ & $81 \mathrm{~b}$ \\
\hline TPC 00607 & 45,6 a & $38,3 \mathrm{~b}$ & 149 a & 92 a \\
\hline S. Sweet & 45,1 a & $34,2 \mathrm{~b}$ & 143 a & 88 a \\
\hline TPR 91960 & $39,9 \mathrm{~b}$ & $18,6 \mathrm{c}$ & $130 \mathrm{a}$ & $60 \mathrm{c}$ \\
\hline Legend & $38,1 \mathrm{~b}$ & $34,1 \mathrm{~b}$ & $141 \mathrm{a}$ & $94 a$ \\
\hline EX-07595001 & $29,5 \mathrm{~b}$ & $27,2 \mathrm{c}$ & $104 \mathrm{~b}$ & $94 a$ \\
\hline CV $(\%)$ & 16,6 & 16,5 & 26,4 & 12,3 \\
\hline
\end{tabular}

Médias seguidas de mesma letra nas colunas não diferem entre si, pelo teste Scott-Knott, a $5 \%$ de probabilidade (Average values followed from the same letter in the column did not differ through the Scott-Knott test, 5\% probability).

como mais produtivas. Resende et al. (2005) obtiveram produtividades totais de bulbos entre 19,1 a $45,1 \mathrm{t} \mathrm{ha}^{-1}$ entre diferentes genótipos, apresentando-se o genótipo Legend como o menos produtivo com 19,1 t ha-1. A produtividade comercial oscilou entre 15,6 a 43,1 $\mathrm{t} \mathrm{ha}^{-1}$, sobressaindo-se os genótipos IPA-11, Superex, Sawana Sweet, EX-07595002, Brisa, TPR 91970, EX-19013 e EX07592000, como os que apresentaram maiores produtividades. $\mathrm{O}$ presente trabalho objetivou identificar genótipos de cebola mais adaptados que apresentem alto potencial para produção e que atendam o mercado consumidor nacional nas condições do Vale do São Francisco.

\section{MATERIAL E MÉTODOS}

Os experimentos foram conduzidos em campo da Embrapa Semi-Árido em Petrolina, de abril a agosto de 2003 e 2004; cujas coordenadas geográficas do local são 09 09' de latitude Sul e $40^{\circ}$ 22 ' de longitude Oeste e altitude de 365,5 m (Amorim Neto, 1989) e o solo classificado como Latossolo Vermelho Amarelo Distróferrico (Embrapa, 1999).

O delineamento experimental utilizado foi em blocos ao acaso, com quin- ze genótipos (Sawana Sweet, TPC 91923; TPR 91970; TPC 00607; LEGEND; TPR 91960; IPA-11; Encino; EX-07593000; EX-07595001; EX07592000; EX-19013; EX-07595002; Superex e Brisa) em três repetições em 2003, e quatorze genótipos (IPA-03, IPA-04, IPA-06, IPA-08, IPA-10, IPA11, Brisa, Texas Grano 502, Texas Grano 502 PRR, Red Creole, Granex 429, Alfa São Francisco ciclo IV, EX-19013 e EX07593000) em quatro repetições em 2004.

Em sementeira semeou-se $10 \mathrm{~g}$ de sementes de cada genótipo por $\mathrm{m}^{2}$. $\mathrm{O}$ transplantio foi efetuado aos 35 dias após a semeadura, em parcelas de 0,60 $\mathrm{m}$ de largura, por 6,0 $\mathrm{m}$ de comprimento com área útil de $3,60 \mathrm{~m}^{2}$, que comportavam quatro linhas de cebolas, no espaçamento de $0,15 \mathrm{~m}$ x 0,20 m.

As irrigações foram feitas por microaspersão com turno de dois dias. Utilizou-se adubo orgânico, esterco de curral curtido, na dose de $10 \mathrm{~L}$ por 3,60 $\mathrm{m}^{2}$. Foram aplicados em fundação 180 $\mathrm{kg} \mathrm{ha}^{-1}$ de $\mathrm{P}_{2} \mathrm{O}_{5}$ e $20 \mathrm{~kg} \mathrm{ha}^{-1}$ de $\mathrm{N}$ (uréia), e em cobertura $130 \mathrm{~kg} \mathrm{ha}^{-1}$ de $\mathrm{N}$, juntamente com o potássio (cloreto de potássio) na dose de $160 \mathrm{~kg} \mathrm{ha}^{-1}$ de $\mathrm{K}_{2} \mathrm{O}$ aos 20; 30; 40 e 50 dias após o transplantio.
Os fertilizantes foliares foram aplicados através de pulverizações semanais, na dose de $50 \mathrm{cc} / 20 \mathrm{~L}$ de água. Os tratos culturais e o controle fitossanitário foram os mesmos utilizados nos plantios convencionais.

A colheita foi realizada quando em média $90 \%$ das plantas encontravam-se tombadas (estalo), conforme o ponto de colheita de cada genótipo. As plantas colhidas foram submetidas ao processo de cura, ficando por seis dias expostas ao sol e dois dias à sombra, efetuandose, em seguida, o corte da parte aérea. Avaliou-se a produtividade total $\left(\mathrm{t} \mathrm{ha}^{-1}\right)$ e comercial ( $\mathrm{t} \mathrm{ha}^{-1}$ ), sendo considerados como comerciais os bulbos com diâmetros maior que $35 \mathrm{~mm}$ (Costa et al., 2000), sendo os bulbos menores (diâmetro transversal inferior a $35 \mathrm{~mm}$ ) computados apenas na produtividade total; massa fresca de bulbo comerciais (g) e número de bulbos comerciais. Os dados foram submetidos à análise de variância, sendo as médias comparadas pelo teste de Scott-Knott ao nível de 5\% de probabilidade (Scott \& Knott, 1974).

\section{RESULTADOS E DISCUSSÃO}

A produtividade total de bulbos em 2003 (Tabela 1) variou de 29,5 a 59,6 t $\mathrm{ha}^{-1}$, destacando-se os genótipos TPR 91970 (59,6 t ha-1), EX-19013 (58,6 t $\left.\mathrm{ha}^{-1}\right)$, EX-07595002 (52,3 $\left.\mathrm{t} \mathrm{ha}^{-1}\right)$, TPC 91923 (52,2 t ha-1), Encino (51,6 t ha-1), EX-07593000 (50,1 t ha-1), sem apresentarem diferenças significativas entre si. Estes resultados superam os obtidos por Resende et al. (2005) no Vale do São Francisco, que verificaram produtividades totais de bulbos entre 19,1 e 45,1 t $\mathrm{ha}^{-1}$, de diferentes genótipos.

A produtividade comercial oscilou de 18,6 a 51,7 t ha-1, sobressaindo-se os genótipos EX-19013 (51,7 t ha-1), EX0759002 (48,6 $\left.\mathrm{t} \mathrm{ha}^{-1}\right)$, IPA-11 (47,6 $\left.\mathrm{t} \mathrm{ha}^{-1}\right)$, Brisa (46,6 t ha-1), TPC 91923 (45,5 t $\left.\mathrm{ha}^{-1}\right)$, TPR 91970 (45,2 $\left.\mathrm{t} \mathrm{ha}^{-1}\right)$, Encino $\left(48,3 \mathrm{t} \mathrm{ha}^{-1}\right)$ e EX-075593000 (42,2 $\left.\mathrm{t} \mathrm{ha}^{-1}\right)$ com as maiores produtividades sem diferirem entre si (variações de 42,2 a 51,7 $\mathrm{t} \mathrm{ha}^{-1}$ ). Estes genótipos apresentaram incrementos na produtividade variando entre $135,8 \%$ e $188,8 \%$, superiores à média nacional de 17,9 t/ha. Esses resultados são superiores aos obtidos por 
Vidigal et al. (2002), que obtiveram produtividades variando de 13,3 a 32,9 tha${ }^{1}$, todavia, inferiores aos obtidos por Costa et al. (2000) e Resende et al. (2003). Os genótipos TPR 91960 e EX07595001 apresentaram os menores desempenhos com 18,6 e 27,2 $\mathrm{t} \mathrm{ha}^{-1}$ de bulbos.

A massa fresca do bulbo variou de 104 a 186 g, não ocorrendo diferenças estatísticas entre os genótipos avaliados, com exceção do Ex-07595001, que apresentou a menor massa de bulbo (104 g bulbo ${ }^{-1}$ ). Esses resultados são similares aos obtidos por Resende et al. (2005), que obtiveram massas frescas dos bulbos de 119,33 a 221,00 g sobressaindose os genótipos Ex-07593001, IPA-11, EX-07595002 e Superex com as maiores massas.

Os genótipos IPA-11 e Brisa apresentaram maior número de bulbos comerciais por parcela (113 bulbos), sem diferirem estatisticamente dos demais, exceto os genótipos Superex, EX07592000 e TPR 91960, que apresentaram menor número de bulbos (Tabela 1). Resende et al. (2005) relatam um número de bulbos comerciais por parcela pouco abaixo dos encontrados no presente trabalho, oscilando entre 84,0 e 101,0 bulbos.

No ano 2004 (Tabela 2), obteve-se produtividade total de bulbos de 30,4 a $58,4 \mathrm{t} \mathrm{ha}^{-1}$, destacando-se os genótipos EX-07593000 (58,4 t ha $\left.{ }^{-1}\right)$, Granex 429 $\left(58,1 \mathrm{t} \mathrm{ha}^{-1}\right)$, IPA-11 (54,5 t ha-1), IPA-4 $\left(50,8 \mathrm{t} \mathrm{ha}^{-1}\right)$ e Texas Grano 502 (50,1 t/ ha), sem diferença estatística entre si. Estes resultados estão dentro da faixa de produtividade total informada por Costa et al. (2002), que obtiveram 41,3 a 74,7 t/ha de bulbos.

A produtividade comercial oscilou de 24,0 a 55,2 $\mathrm{t} \mathrm{ha}^{-1}$, sobressaindo os genótipos Granex 429 (56,0 t ha-1), EX$07593000\left(55,2 \mathrm{t} \mathrm{ha}^{-1}\right)$, IPA-11 (51,3 $\left.\mathrm{t} \mathrm{ha}^{-1}\right)$, IPA-4 (47,9 tha $\left.^{-1}\right)$, Texas Grano 502 (47,1 $\left.\mathrm{t} \mathrm{ha}^{-1}\right)$ e Brisa $\left(41,8 \mathrm{t} \mathrm{ha}^{-1}\right)$ como os mais produtivos. Estes genótipos obtiveram incrementos na produtividade, variando de 134,1 a 208,4\% superiores à média nacional. Estes resultados se assemelham aos rendimentos obtidos por Duarte et al. (2003), entre 40,5 e 43,7 t ha-1, e dentro da faixa dos resultados encontrados por Costa et al. (2000), com produtividade

Tabela 2. Produtividade total e comercial, massa fresca de bulbos e número de bulbos comerciais por parcela de genótipos de cebola (Commercial and total yield, fresh mass of bulbs and number of commercial bulbs in each parcel of onion genotypes).

\begin{tabular}{|c|c|c|c|c|}
\hline \multirow{2}{*}{ Genótipos } & \multicolumn{2}{|c|}{ Produtividade $\left(\mathrm{t} \mathrm{ha}^{-1}\right)$} & \multirow{2}{*}{$\begin{array}{l}\text { Massa fresca } \\
\text { do bulbo }(\mathrm{g})\end{array}$} & \multirow{2}{*}{$\begin{array}{c}\mathrm{N}^{\circ} \\
\text { bulbos/parcela }\end{array}$} \\
\hline & Total & Comercial & & \\
\hline EX-07593000 & $58,4 \mathrm{a}$ & $55,2 \mathrm{a}$ & $145 b$ & $126 \mathrm{a}$ \\
\hline Granex 429 & $58,1 \mathrm{a}$ & 56,0 a & $136 \mathrm{~b}$ & $124 \mathrm{a}$ \\
\hline IPA -11 & 54,5 a & 51,3 a & $135 b$ & $115 b$ \\
\hline IPA - 04 & 50,8 a & $47,9 \mathrm{a}$ & $145 b$ & $98 \mathrm{c}$ \\
\hline Texas Grano 502 & $50,1 \mathrm{a}$ & $47,1 \mathrm{a}$ & $160 \mathrm{a}$ & $89 c$ \\
\hline Brisa & $44,8 \mathrm{~b}$ & $41,8 \mathrm{~b}$ & $116 \mathrm{c}$ & $114 \mathrm{~b}$ \\
\hline Alfa São Francisco & $42,6 \mathrm{~b}$ & $38,9 \mathrm{~b}$ & $129 b$ & $90 \mathrm{c}$ \\
\hline IPA - 10 & $42,2 \mathrm{~b}$ & $38,3 \mathrm{~b}$ & $108 \mathrm{c}$ & $108 b$ \\
\hline IPA - 03 & $41,0 \mathrm{~b}$ & $36,7 \mathrm{~b}$ & $159 a$ & $80 \mathrm{~d}$ \\
\hline IPA - 08 & $39,6 \mathrm{~b}$ & $33,9 \mathrm{~b}$ & $96 \mathrm{~d}$ & $106 \mathrm{~b}$ \\
\hline Texas Grano 502 PRR & $38,9 \mathrm{~b}$ & $35,9 \mathrm{~b}$ & $136 \mathrm{~b}$ & $79 d$ \\
\hline EX - 19013 & $35,4 \mathrm{c}$ & $33,8 \mathrm{~b}$ & $131 \mathrm{~b}$ & $78 \mathrm{~d}$ \\
\hline IPA - 06 & $35,2 \mathrm{c}$ & $31,5 \mathrm{~b}$ & $95 \mathrm{~d}$ & $94 \mathrm{c}$ \\
\hline Red Creole & $30,4 \mathrm{c}$ & $24,0 \mathrm{c}$ & $86 \mathrm{~d}$ & $81 \mathrm{~d}$ \\
\hline C.V. (\%) & 10,8 & 12,8 & 9,3 & 7,5 \\
\hline
\end{tabular}

Médias seguidas de mesma letra nas colunas não diferem entre si, pelo teste Scott-Knott, a $5 \%$ de probabilidade (Average values followed from the same letter in the column did not differ through the Scott-Knott test, $5 \%$ probability).

entre 34,8 e $61,1 \mathrm{t} \mathrm{ha}^{-1}$ de bulbos comerciais.

A massa fresca de bulbo variou de 86 a $160 \mathrm{~g}$, destacando-se Texas Grano 502 (160 $\left.\mathrm{g} \mathrm{bulbo}^{-1}\right)$ e IPA-3 (159 g bul$\left.\mathrm{bo}^{-1}\right)$; a pior performance foi apresentada pelos genótipos IPA-08, IPA-06 e Red Creole, não diferindo entre si. Estes resultados são inferiores àqueles obtidos por Resende et al. (2005), que verificaram massa fresca do bulbo entre 119,33 e $221,00 \mathrm{~g}$.

Os genótipos EX-07593000 e Granex 429 apresentaram maior número de bulbos comerciais por parcela (126 e 124 bulbos, respectivamente), sendo o pior desempenho obtido pelos genótipos IPA-03, Texas Grano 502 PRR, EX-19013 e Red Creole com variações de 78 a 81 bulbos parcela ${ }^{-1}$. Esses resultados são comparáveis aos obtidos por Resende et al. (2005) que observaram variações no número de bulbos comerciais por parcela entre 84,0 e 101,0 bulbos.

O mercado consumidor nacional prefere bulbos de tamanho médio com massa entre 80 e 150 gramas (Souza \& Resende, 2002; May, 2006); neste contexto, os genótipos avaliados obtiveram massas frescas de bulbo na faixa adequada ou pouco acima da preferência do consumidor nacional, fato este que pode provavelmente estar associado ao espaçamento adotado de 0,20 x 0,15 m, mas que pode ser sanado pelo uso de maiores densidades de plantio, conforme se pode observar em pesquisas realizadas por diversos autores (Candeia et al., 2001; Reghin et al., 2003; May, 2006).

As maiores produtividades, massas frescas e número de bulbos comerciais obtidos pelos melhores genótipos devem-se provavelmente à sua melhor adaptação às condições edafoclimáticas regionais. Quando as condições climáticas não satisfazem as exigências do genótipo, especialmente o comprimento do dia (fotoperíodo) e temperatura, poderá ocorrer a não formação de bulbos, proporcionando um elevado índice de plantas improdutivas "charutos"; emissão de pendão floral e formação de bulbos pequenos (Galmarini, 1997), com conseqüente baixa produtividade. Neste contexto, depreende-se pelos resultados das características avaliadas, que os genótipos EX-19013, EX07595002, Encino, IPA-11, Brisa, TPC 91923, TPR 91970 e EX-075593000 (em 2003) e os genótipos EX-0759300, Granex 429, IPA-11, IPA-04 e Texas Grano 502 (em 2004), apresentam-se 
como os mais adaptados e com maior potencial para cultivo nas condições do Vale do São Francisco. No entanto, justifica-se a necessidade de maiores pesquisas no sentido de estudar diferentes densidades de plantio que promovam menor diâmetro do bulbo, adequando estes à preferência do mercado consumidor nacional.

\section{REFERÊNCIAS}

AMORIM NETO MS. 1989. Informações meteorológicas dos Campos Experimentais de Bebedouro e Mandacaru, Petrolina, PE. Petrolina: EMBRAPA - CPATSA. 58p. (EMBRAPA - CPATSA. Documentos, 57).

CANDEIA JA; MENEZES D; SANTOS VF; MENEZES JT. 2001. Desempenho agronômico de cultivares de cebola em Pernambuco. Horticultura Brasileira 19, Suplemento. CDROM. (Trabalho apresentado no $41^{\circ}$ Congresso Brasileiro de Olericultura, 2001).

COSTA ND; RESENDE GM; DIAS RCS. 2000 Avaliação de cultivares de cebola em PetrolinaPE. Horticultura Brasileira 18: 57-60.
COSTA ND; LEITE DL; SANTOS CAF; FARIA; CANDEIA JA; VIDIGAL SM. 2002. Cultivares de cebola. Informe Agropecuário 23: 20-27.

DUARTE RLR; VELOSO MEC; MELO FB; ATHAIDE SOBRINHO C; RIBEIRO VQ; SILVA PHS. 2003. Produtividade de cultivares de cebola no Semi-Árido piauiense. Horticultura Brasileira 21: 34-36.

EMPRESA BRASILEIRA DE PESQUISA AGROPECUÁRIA. 1999. Sistema brasileiro de classificação de solos. Brasília: Embrapa Produtividade de Informações (SPI). 412 p.

FAO. Agricultural production, primary crops. Disponível em <http://www.fao.org>. Acesso em: 17 out. 2006.

GALMARINI CR; GASPERA PG. 1995. Efecto de la epoca de transplante y la densidad de plantacion en el cultivo de cebolla tipo Valenciana. Horticultura Argentina 14: 23-29.

INSTITUTO BRASILEIRO DE GEOGRAFIA E ESTATÍSTICA - IBGE. Produção Agrícola Municipal. Disponível em <http:// www.sidra.ibge.gov.br>. Acesso em: 26 jan. 2005.

MAY A. 2006. Desempenho de híbridos de cebola em função da população de plantas e fertilização nitrogenada e potássica. Jaboticabal: UNESP- FCAV, 142p. (Tese Doutorado).
REGHIN MY; OTTO RF; JACOBY CFS; OLINIK JR; OLIVEIRA RP. 2004. Efeito da densidade de plantas no rendimento de bulbos com diferentes cultivares de cebola. Horticultura Brasileira 22, Suplemento 2. CDROM. (Trabalho apresentado no $44^{\circ}$ Congresso Brasileiro de Olericultura, 2004).

RESENDE GM; CHAGAS SJR; PEREIRA LV. 2003. Característica produtivas de cultivares de cebola no Sul de Minas Gerais. Horticultura Brasileira 21: 722-725.

RESENDE GM; COSTA ND; SANTOS CAF; SANTOS GM; LEITE WM. 2005. Desempenho produtivo de genótipos de cebola em Vertissolo no Vale do São Francisco. Caatinga 18: 210-214.

SCOTT AJ; KNOTT M. 1974. A Cluster analysis method for grouping means in the analysis of variance. Biometrics 30: 507-512.

SOUZA RJ; RESENDE GM. 2002. Cultura da cebola. Lavras: Editora UFLA, 115p. (UFLA, Textos Acadêmicos, 21).

VIDIGAL SM; FACION CE; PACHECO DD 2002. Avaliação de cultivares de cebola na região Norte de Minas Gerais. Horticultura Brasileira 20. Suplemento 2. CD-ROM. Edição de Resumos do $42^{\circ}$ Congresso Brasileiro de Olericultura, Uberlândia-MG. 